\title{
Semen quality, testicular B-mode and Doppler ultrasound, and serum testosterone concentrations in dogs with established infertility
}

\author{
Mírley Barbosa de Souza ${ }^{\mathrm{a}, *}$, Gary C.W. England ${ }^{\mathrm{b}}$, \\ Antônio Cavalcante Mota Filho a ${ }^{a}$, Camila Louise Ackermann ${ }^{c}$, \\ Carmen Vládia Soares Sousa a , Gabriela Guedelha de Carvalho a, \\ Herlon Victor Rodrigues Silva a, José Nicodemos Pinto ${ }^{a}$, \\ Jussiara Candeira Spíndola Linhares ${ }^{\mathrm{d}}$, Eunice Oba ${ }^{\mathrm{c}}$, \\ Lúcia Daniel Machado da Silva ${ }^{a}$

\footnotetext{
a Laboratory of Carnivore Reproduction, Veterinary School, State University, Fortaleza, Ceará, Brazil

${ }^{\mathrm{b}}$ School of Veterinary Medicine and Science, The University of Nottingham, Loughborough, UK

${ }^{\mathrm{c}}$ Department of Animal Reproduction and Veterinary Radiology, Faculty of Veterinary Medicine and Animal Science, Universidade Estadual Paulista Júlio de Mesquita Filho, Botucatu, São Paulo, Brazil
} \\ ${ }^{\mathrm{d}}$ Federal University of Piauí, Amílcar Ferreira Sobral Campus, Floriano, Piauí, Brazil
}

\section{A R T I C L E I N F O}

\section{Article history:}

Received 15 August 2014

Received in revised form 19 May 2015

Accepted 19 May 2015

\section{Keywords:}

Ultrasonography

Pulse-wave Doppler

Dog

Testis

Infertility

\begin{abstract}
A B S T R A C T
Retrospective examination of breeding records enabled the identification of 10 dogs of normal fertility and 10 dogs with established infertility of at least 12 months of duration. Comparisons of testicular palpation, semen evaluation, testicular ultrasound examination, Doppler ultrasound measurement of testicular artery blood flow, and measurement of serum testosterone concentration were made between the two groups over weekly examinations performed on three occasions. There were no differences in testicular volume $\left(\mathrm{cm}^{3}\right)$ between the two groups (fertile right testis $=10.77 \pm 1.66$; fertile left testis $=12.17 \pm 2.22$ ); (infertile right testis $=10.25 \pm 3.33$; infertile left testis $=11.37 \pm 3.30$ ), although the infertile dogs all had subjectively softer testes compared with the fertile dogs. Infertile dogs were either azoospermic or when they ejaculated, they had lower sperm concentration, sperm motility, and percentage of morphologically normal spermatozoa than fertile dogs. Furthermore, infertile dogs had reduced sperm membrane integrity measured via the hypoosmotic swelling test. Infertile dogs had significantly lower basal serum testosterone concentrations $(1.40 \pm 0.62 \mathrm{ng} / \mathrm{mL})$ than fertile dogs $(1.81 \pm 0.87 \mathrm{ng} / \mathrm{mL}$; $\mathrm{P}<0.05)$. There were subjective differences in testicular echogenicity in some of the infertile dogs, and important differences in testicular artery blood flow with lower peak systolic and enddiastolic velocities measured in the distal supratesticular artery, marginal testicular artery, and intratesticular artery of infertile dogs $(\mathrm{P}<0.05)$. Notably, resistance index and pulsatility index did not differ between infertile and fertile dogs. These findings report important differences between infertile and fertile dogs which may be detected within an expanded breeding soundness examination.
\end{abstract}

(C) 2015 Elsevier Inc. All rights reserved.

\footnotetext{
* Corresponding author. Tel.: +55 853101 9851; fax: +55 8531019840 E-mail address: mirley.souza@gmail.com (M.B. de Souza).
}

\section{Introduction}

Conducting a breeding soundness examination (BSE) is a well-established method for evaluating the breeding potential of dogs [1]. The principle of the BSE is that it may 
detect features predictive of poor breeding or fertilizing potential, but despite the wide recommendation for use of the BSE [2], there have been no comprehensive studies examining differences in BSE between fertile and infertile dogs. There are lamentably few investigations comparing even individual components of the BSE between fertile and infertile dogs; the most significant study was performed more than 20 years ago and compared only sperm morphology [3]. More recent and elaborate investigation, for example of sperm DNA peroxidate, has found no differences between infertile and fertile dogs [4].

The key aspects of a BSE include clinical examination of the reproductive tract, observation of libido, examination of semen quality, and in some cases ultrasound examination of the reproductive tract, and endocrine testing [1,2]. More recently, measurement of testicular artery flow has been purported to be of some value [5] and may form part of an expanded BSE although data are available from only a small number of individuals [5].

The study aim was to establish which aspects of an expanded BSE were different between known fertile and known infertile dogs.

\section{Materials and methods}

\subsection{Animals}

This study was performed in the Laboratory of Carnivore Reproduction at the School of Veterinary Medicine, State University of Ceará and approved by the Animal Ethics Committee of the institution (protocol 12641034-8).

Animals were selected on the basis of evaluation of detailed breeding records from private breeders who had meticulous records, and two groups were identified. Fertile dogs comprised 10 dogs that had mated at least four bitches during the previous 12 months each achieving at least two normal pregnancies with a normal litter size for the breed [6]. Infertile dogs comprised 10 dogs that had mated at least four bitches during the previous 12 months with no resultant pregnancy. All bitches had been previously pregnant, and in both groups, they were bred by natural mating at a time identified by vaginal cytology and measurement of plasma progesterone concentration.

The fertile dogs comprised Labrador $(n=2)$, Rottweiler $(\mathrm{n}=4)$ and German Shepherd $(\mathrm{n}=4)$ breeds, ranging from 2 to 8 years old (mean \pm standard deviation $=4.5 \pm 1.9$ ), weighing 33 to $42 \mathrm{~kg}$. The infertile dogs comprised Fila Brasileiro $(\mathrm{n}=2)$, Golden Retriever $(n=2)$, Rottweiler $(n=3)$, and German Shepherd $(\mathrm{n}=3)$ breeds, aged from 4 to 8 years (mean \pm standard deviation $=5.4 \pm 1.4$ ), weighing 35 to $44 \mathrm{~kg}$.

Veterinary clinical examination and complete blood count at the beginning of the study confirmed that all dogs were clinically normal and healthy. All dogs were fed a maintenance complete dry food with ad libitum water for the duration of the study.

\subsection{Breeding soundness examination}

Each dog was subject to all aspects of the BSE on three occasions at 7-day intervals. The fertile or infertile status of the dog was not known by the evaluator. At each examination, the scrotal contents were palpated and a subjective assessment of the testes consistency was made which was recorded as firm or soft. Ejaculates were then collected from each dog by digital manipulation, and the second fraction of the ejaculate was immediately subjected to detailed examination. The second fraction volume was recorded, and a subjective microscopic assessment of the percentage total sperm motility [7] was made at $\times 400$ magnification at room temperature. Sperm concentration was measured using a Neubauer chamber after dilution with formal saline [8], and sperm morphology was evaluated at $\times 1000$ magnification on rose bengal-stained slides [3]. Membrane integrity was evaluated at $\times 400$ magnification using the hypoosmotic swelling test (HOST) [9].

Ultrasound examinations were performed on the right and left testis of each dog with 7-day intervals using a SonoAce PICO machine (Medison, Korea) with a lineararray transducer with 5- to 9-MHz capability. Dogs were positioned in dorsal recumbency, acoustic gel was applied to the skin, and the transducer was positioned initially on the lateral surface of the testis. Longitudinal and transverse B-mode images were made (using the mediastinum as a reference point for measuring the testicular length and width), and testicular volume was calculated using the formula for an ellipsoid; $\mathrm{V}=$ length $\times$ width $\times$ height $\times$ 0.5236 . The appearance of the parenchyma of each testis was recorded subjectively as normal echogenicity, hypoechoic, or hyperechoic. In addition, the presence of abnormal echogenic stippling was recorded as present or absent.

For the measurement of testicular artery flow in three separate regions, color Doppler ultrasound was used with the transducer initially placed at the neck of the scrotum to identify the tortuous distal (looping) region of the supratesticular artery (here termed distal supratesticular artery), immediately cranial to the cranial pole of the testis. The transducer was then moved distally to identify the marginal region in longitudinal section (here termed marginal testicular artery), and the relatively straight intratesticular arteries within the testicular parenchyma (here termed intratesticular arteries). The proximal region of the supratesticular artery was not studied because it was not possible to ensure consistency of position between dogs. Within each region, the color gain was adjusted to reduce any excess color noise and the pulse-wave Doppler gate was positioned within the lumen of the vessel. Three waves of a cardiac cycle were used to measure mean values for peak systolic velocity (PSV), end-diastolic velocity (EDV), and these were used by the machine software to calculate resistance index (RI) and pulsatility index (PI). The operator and machine presets (depth, 4.5-5.5 cm; pulsed repetition frequency, $2.5 \mathrm{kHz}$; wall filter, $5 \mathrm{~cm} / \mathrm{s}$; sample gate, $2.0 \mathrm{~mm}$ ) were consistent for each region at all examinations. The angle between the Doppler beam and the long axis of the vessel was less than $60^{\circ}$, using angle corrections when necessary. However, in most cases, an angle of $0^{\circ}$ was used.

Blood was collected from each dog weekly on three occasions at 9 AM each day. After clotting, serum was harvested and frozen at $-80{ }^{\circ} \mathrm{C}$ until evaluation for testosterone concentration using a commercially available radioimmunoassay kit (Total Testosterone Coat-A-Count; 
Diagnostics Products Corporation, Los Angeles, CA, USA). The intra-assay and interassay coefficients of variation were $1.28 \%$ and $5.9 \%$, respectively.

\subsection{Statistical analysis}

Data were tested for normality (Shapiro-Wilk test) and homoscedasticity (Levene test). A two-factor ANOVA was used to test for differences in testicular volume between weeks, right and left testis volume, and to investigate differences between the fertile and infertile dogs.

Semen quality data were submitted to the Friedman test to compare values between the weeks of evaluation and to the Mann-Whitney test for comparison between fertile and infertile dogs.

Doppler ultrasound parameters were compared using a two-factor ANOVA to test for differences between regions of the testes, right and left testes, and between fertile and infertile dogs, using weeks of evaluation as one of the factors.

Two-factor ANOVA was used to examine differences between weeks and groups for serum testosterone concentrations. A significance level of $\mathrm{P}<0.05$ was used in all cases, and the results were expressed as the mean \pm standard deviation.

To investigate any relations between intratesticular artery flow, coefficients of correlation were calculated between each of the flow measurements (PSV, EDV, RI, PI) and each of the semen quality measurements (total motility, sperm concentration, HOST, morphologically normal sperm) for all 20 dogs. The Pearson productmoment correlation coefficient was calculated, and significant correlations were considered significant when $\mathrm{P}<0.05$.

\section{Results}

The testes of all fertile dogs were reported as being firm in texture, whereas the testes from all infertile dogs were reported as soft in texture. No other scrotal abnormalities were noted.

The second fraction of the ejaculate from the fertile dogs had a white opaque appearance, whereas for the infertile dogs, the ejaculates were colorless in five dogs (these samples were confirmed as azoospermic) and watery white in the remaining five dogs. Semen quality did not differ between the weeks of evaluation, and none of the

\section{Table 1}

Mean \pm standard deviation semen quality parameters of the second fraction of the ejaculate of 10 fertile dogs collected on three occasions $(n=30)$ and five infertile dogs collected on three occasions $(n=15)$.

\begin{tabular}{lcc}
\hline Seminal parameters & Fertile dogs & Infertile dogs $^{\mathrm{c}}$ \\
\hline Second fraction volume $(\mathrm{mL})$ & $0.91 \pm 0.60^{\mathrm{a}}$ & $0.65 \pm 0.26^{\mathrm{a}}$ \\
Total motility $(\%)$ & $96.0 \pm 4.8^{\mathrm{a}}$ & $71.0 \pm 14.0^{\mathrm{b}}$ \\
Sperm concentration $\left(\times 10^{6} / \mathrm{mL}\right)$ & $1295.0 \pm 1153.2^{\mathrm{a}}$ & $50.00 \pm 530.3^{\mathrm{b}}$ \\
Hypoosmotic swelling test & $93.2 \pm 2.9^{\mathrm{a}}$ & $88 \pm 3.7^{\mathrm{b}}$ \\
Normal spermatozoa $(\%)$ & $89 \pm 9.7^{\mathrm{a}}$ & $70.13 \pm 8.3^{\mathrm{b}}$ \\
\hline
\end{tabular}

${ }^{\mathrm{a}, \mathrm{b}}$ Different lowercase letters represent significant between the fertile and infertile dogs $(\mathrm{P}<0.05)$.

c Ten infertile dogs were included in the study but five were azoospermic and were not included in this table.

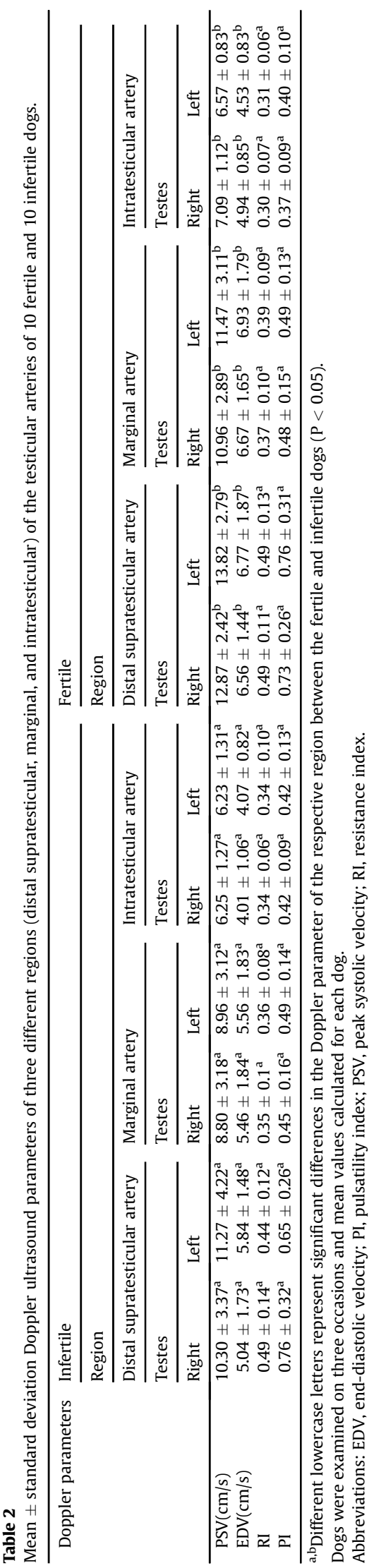


azoospermic dogs produced an ejaculate containing sperm. Total sperm motility, sperm concentration, percentage of swollen sperm in the HOST, and the percentage of normal spermatozoa were higher in fertile compared with infertile dogs that produced sperm, although there were no differences in the ejaculate volume (Table 1).

Testicular volume did not differ between the weeks of evaluation for either group. Testicular volume $\left(\mathrm{cm}^{3}\right)$ was not different between the fertile (right testis $=10.77 \pm 1.66$; left testis $=12.17 \pm 2.22$ ) and infertile dogs (right testis $=10.25 \pm 3.33$; left testis $=11.37 \pm 3.30$ ), although for each group, the left testes had a significantly greater volume $(\mathrm{P}<0.05)$.

Subjective scoring of testicular echogenicity of the fertile dogs showed that eight had bilateral normal echogenicity testes, one had bilateral normal echogenicity testes with echogenic stippling in one testes, whereas one dog had bilateral hypochoic testes. For the infertile dogs, two had normal echogenicity testes, four had bilateral hypochoic testes, two had bilateral hyperechoic testes, and two had bilateral hyperechoic testes with echogenic stippling in one testis.

Color Doppler allowed identification of all regions of the testicular artery of the left and right testes of all dogs. The distal supratesticular artery had a tortuous pattern along its entire length, and although it was possible to visualize the artery in both groups, it subjectively appeared less tortuous and it was more difficult to capture the color Doppler signal in infertile dogs. The marginal testicular artery had a linear pattern and was observed along the entire length of the testis and did not appear different between either group. The intratesticular arteries were visible throughout the testicular parenchyma of both groups, following a linear pattern directed toward the mediastinum testis.

When visualized by pulse-wave Doppler, the waveforms of the testicular artery blood flow in fertile dogs, within the supratesticular region, were biphasic with a diastolic notch followed by a diastolic peak in four dogs and monophasic with systolic peaks, decreasing diastolic flow and low vascular resistance in six dogs. Testicular artery blood flow within the marginal and intratesticular region was monophasic for all dogs. For the infertile dogs, the waveforms had a more venous-like waveform appearance in the three regions, with lower velocities than for the fertile dogs. This flow pattern was differentiated from venous flow which could also be identified. Images for color and pulse-wave Doppler for fertile and infertile dogs are provided in the Supplemental Material.

Doppler measurements did not differ between the weeks of evaluation or between the right and left testes for either group. Similarly, for either group, there were no regional differences in PSV, EDV, RI, or PI, although each of these parameters was numerically greater in the distal supratesticular region (Table 2).

Correlations between the Doppler arterial flow measurements of the intratesticular arteries and semen quality for all 20 dogs showed that there was a significant negative correlation between $\mathrm{RI}$ and total motility $(r=-0.30 ; \mathrm{P}=0.05)$ and between PI and total sperm motility $(r=-0.37 ; \mathrm{P}=0.01)$. There were no other significant correlations.
Serum testosterone concentrations in the fertile and infertile dogs did not differ between the weeks. Serum testosterone concentrations were significantly higher in the fertile dogs $(1.81 \pm 0.87 \mathrm{ng} / \mathrm{mL})$ compared with the infertile dogs $(1.40 \pm 0.62 \mathrm{ng} / \mathrm{mL})$.

\section{Discussion}

The central principle of the BSE is that particular components may be useful for the differentiation of normal from abnormal males. In this study, a group of dogs of known fertile status were compared with a group that had failed to achieve any pregnancies over the preceding 12 months.

It was interesting that ultrasound-measured testicular volume did not differ between the fertile and infertile dogs, similar to observations previously made in the dog [10] and in men [11], llamas and alpacas [12]. It is clear that testicular volume alone is not a reliable parameter for evaluating dogs with a history of infertility; however, softening of the testes detected by palpation was reported in all infertile but none of the fertile males in the present study, suggesting that this can be a useful component of the BSE. The relation between testis tone and semen quality has previously been remarked on dogs [3], but this is the first report that evidences softening of the testes as a significant feature of infertility.

Evaluation of a semen sample is an important aspect of the BSE [13]. In this study, semen quality measurements from the fertile dogs were similar to those previously reported [7,14]. Interestingly, but not unexpectedly, five of the infertile dogs were azoospermic, and for the remaining infertile dogs which produced sperm, there were lower values of total sperm motility, sperm concentration, morphologically normal sperm, and sperm membrane integrity evaluated using the HOST, compared with the fertile dogs. Interestingly, although the HOST has been adequately described in dogs [9,15], there has been limited study of hypoosmotic swelling of sperm in known infertile dogs. Our study is a useful addition to the literature in this area, especially since the validity of the HOST has recently been questioned [16].

In the present study, B-mode ultrasound imaging of the testicular parenchyma of the fertile dogs showed a subjective appearance similar to that previously reported [10], although interestingly one fertile dog had echogenic stippling present in one testis, and another had hypoechoic testes; both features that have been reported as abnormal $[10,17]$. Importantly, two infertile dogs had normal echogenicity testes, four had bilateral hypochoic testes, two had bilateral hyperechoic testes, and two had bilateral hyperechoic testes with echogenic stippling in one testis. It is clear that subjective assessment of testicular architecture is difficult to relate to fertile status because both normal appearing and hypoechoic testes were seen in both fertile and infertile dogs. Hyperechoic testes were only seen in infertile dogs.

Using color Doppler, it was possible to identify the distal supratesticular, marginal, and intratesticular artery regions of the testicular artery, similar to that previously reported in dogs $[18,19]$. Blood flow was measured in the three regions at 
each examination, and the distal supratesticular artery was easiest to identify having a tortuous pattern also observed in men [20], stallions [21], and in dogs [5,18,19,22,23]. The marginal region had an appearance similar to that previously reported [5,18,19,23], whereas the intratesticular arteries had a linear pattern directed toward the mediastinum, unlike a report documenting that flow could not be measured in these vessels [22]. In men, studies have shown that the intratesticular arteries were better visualized oblique to the longitudinal and transverse planes [20], which is similar to the imaging plane observed in this study.

Pulse-wave Doppler detected two different normal waveforms in the fertile dogs, similar to previous studies in men [20], camelids [12], and dogs [18,23]. Previous work has reported regional differences in PSV, EDV, RI, or PI with the highest values present within the distal supratesticular artery [24,25]. Similar trends were present in both fertile and infertile dogs in the present study although regional differences were not statistically significant.

In the infertile dogs, waveforms had low peak systolic velocities and appeared more venous-like; PSV and EDV were significantly lower in all regions of the testicular artery in the infertile dogs than in the fertile dogs. Resistance index and PI did not differ between fertile and infertile dogs in any region. The finding that infertile dogs had lower PSV and EDV, but that RI and PI were not different to fertile dogs is interesting because reduced blood flow with no change in the vascular bed resistance can only be mediated by multiple factors. Potentially infertile dogs had smaller diameter and less tortuous vessels, which may allow for reduced flow with no change in RI [26].

The magnitude of decreased PSV and EDV in the testicular artery of infertile dogs was small and may be difficult to document in an individual clinical case. Nevertheless, the features of low testicular artery flow noted in the infertile dogs were similar to those seen in infertile llamas and alpacas [12].

Measurement of testosterone may be useful in a BSE, although generally, frequent samples are needed to account for normal diurnal variation [27]. In this study, serum testosterone concentrations were similar to those previously reported in dogs [27], and interestingly, there were significantly lower concentrations in infertile dogs although these remained in the normal range. Recent studies in man have shown that although diurnal variation of testosterone occurs, if samples are collected at the same time of the day, large variations can be overcome and single samples may be diagnostically useful [28]. From the present study, although differences would be difficult to detect and interpret in clinical practice, it might be postulated that a useful assessment of Leydig cell function can be achieved by a single basal testosterone measurement.

This study provides comprehensive evidence that components of a BSE can be related to fertility in dogs. In particular, detection of testicular softening, changes in some seminal characteristics, increased testicular echogenicity, reduced testicular artery blood flow, and decreased serum testosterone concentrations are associated with infertility, and they should form important components of an expanded BSE.

\section{Acknowledgments}

The authors would like to thank the funding agencies for financial support: CAPES (Coordenação de Aperfeiçoamento de Pessoal de Nível Superior - Brazilian Federal Agency for the Support and Evaluation of Graduate Education), CNPq (Conselho Nacional de Desenvolvimento Científico e Tecnológico - National Council for Scientific and Technological Development) through individual research grants to LDMS (grant number 305420/2013-5) and FUNCAP (Fundação Cearense de Apoio ao Desenvolvimento Científico e Tecnológico - Ceara State Support for Scientific and Technological Development Foundation. The authors also thank 4th Cia de Choque/CPCÃES and private owners for providing the animals used in this experiment.

\section{Appendix A. Supplementary data}

Supplementary data associated with this article can be found, in the online version, at http://dx.doi.org/10.1016/j. theriogenology.2015.05.015.

\section{References}

[1] Memon MA. Common causes of male dog infertility. Theriogenology 2007;68:322-8.

[2] Lopate C. The problem stud dog. Vet Clin North Am Small Anim Pract 2012;42:469-88.

[3] Oettlé EE. Sperm morphology and fertility of the dog. J Reprod Fertil Suppl 1993;47:257-60.

[4] Lopes-Santiago BV, Monteiro GA, Bittencourt R, Arduino F, Ovidio PP, Jordão-Junior AA, et al. Evaluation of sperm DNA peroxidation in fertile and subfertile dogs. Reprod Domest Anim 2012; 6:208-9.

[5] Zelli R, Troisi A, Elad Ngonput A, Cardinali L, Polisca A. Evaluation of testicular artery blood flow by Doppler ultrasonography as a predictor of spermatogenesis in the dog. Res Vet Sci 2013;95: 632-7.

[6] Christiansen IBJ. Reproduction in the dog and cat. London: Bailliere Tindall; 1984. p. 181.

[7] England GCW. Semen quality in dogs and the influence of a shortinterval second ejaculation. Theriogenology 1999;52:981-6.

[8] Cardoso RSC, Silva AR, Uchoa DC, Silva LDM. Cryopreservation of canine sêmen using a coconut water extender with egg yolk and three different glycerol concentration. Theriogenology 2003;59: $743-51$.

[9] Quintela AT, Oliveira IRS, Souza AO, Gusmão AL, Silva AR. Waterinduced hypo-osmotic test for the evaluation of canine sperm membrane integrity. Anim Reprod 2010;7:70-4.

[10] England GCW. The relationship between ultrasonographic appearance, testicular size, spermatozoal output and testicular lesions in the dog. J Small Anim Rep 1991;32:306-11.

[11] Pinggera GM, Mitterberger M, Bartsch G, Strasser H, Gradl J, Aigner $\mathrm{F}$, et al. Assessment of the intratesticular resistive index by colour Doppler ultrasonography measurements as a predictor of spermatogenesis. BJU Int 2008;101:722-6.

[12] Kutzler M, Tyson R, Grimes M, Timm K. Determination of testicular blood flow in camelids using vascular casting and color pulsedwave Doppler ultrasonography. Vet Med Int 2011;2011:638602.

[13] Rijsselaere T, Van Soom A, Tanghe S, Coryn M, Maes D, de Kruif A. New techniques for the assessment of canine semen quality: a review. Theriogenology 2005;64:706-19.

[14] England GCW, Allen WE. Fertility and semen parameters in dogs. Vet Rec 1989;125:399.

[15] England GCW, Plummer JM. Hypo-osmotic swelling of dog spermatozoa. J Reprod Fertil Suppl 1993;47:261-70.

[16] Karger S, Arlt S, Haimerl P, Heuwieser W. A systematic review of studies performing the hypo-osmotic swelling test to evaluate the quality of canine spermatozoa. Reprod Domest Anim 2014;49:1-6.

[17] Hetch S. Male reproductive tract. In: Penninck D, D’Anjou MA, editors. Atlas of small animal ultrasonography. Ames, IA: Blackwell Publishing; 2008. p. 417-45. 
[18] Gumbsch P, Holzmann A, Gabler C. Colour-coded duplex sonography of the testes in dogs. Vet Rec 2002;151:140-4.

[19] Carrillo JD, Soler M, Lucas X, Agut A. Colour and pulsed Doppler ultrasonographic study of the canine testis. Reprod Domest Anim 2011;47:655-9.

[20] Middleton WD, Thorne DA, Melson GL. Color Doppler ultrasound of the normal testis. Am J Roentgenol 1989;152:293-7.

[21] Pozor MA, McDonnell SM. Color Doppler ultrasound evaluation of testicular blood flow in stallions. Theriogenology 2004;61:799-810.

[22] Günzel Apel AR, Moè Hrke C, Poulsen Nautrup C. Colour-coded and pulsed Doppler sonography of the canine testis, epididymis and prostate gland: physiological and pathological findings. Reprod Domest Anim 2001;36:236-40.

[23] Souza MB, Barbosa CC, Pereira BS, Monteiro CLB, Pinto JN, Linhares JCS, et al. Doppler velocimetric parameters of the testicular artery in healthy dogs. Res Vet Sci 2014;96:533-6.
[24] Souza MB, Mota Filho AC, Sousa CVS, Monteiro CLB, Carvalho GG, Pinto JN, et al. Triplex Doppler evaluation of the testes in dogs of different sizes. Pesq Vet Bras 2014;34:1135-40.

[25] de Souza MB, Barbosa CC, England GCW, Mota Filho AC, Sousa CVS, Carvalho GG, et al. Regional differences of testicular arterial blood flow in post pubertal and pre-pubertal dogs. BMC Vet Res 2015;11:47.

[26] Helmberger M, Pienn M, Urschler M, Killnig P, Stollberger R, Kovacs $G$, et al. Quantification of tortuosity and fractal dimension of the lung vessels in pulmonary hypertension patients. PLoS One 2014;9:e87515.

[27] Souza FF, Leme DP, Uechi E, Lopes MD. Evaluation testicular fine needle aspiration cytology and serum testosterone levels in dogs. Braz J Vet Res Anim Sci 2004;41:98-105.

[28] Brambilla DJ, Matsumoto AM, Araujo AB, McKinlay JB. The effect of diurnal variation on clinical measurement of serum testosterone and other sex hormone levels in men. J Clin Endocrinol Metab 2009;94:907-13. 